\title{
Thyroid nodules and cancer in children and adolescents affected by Hashimoto's thyroiditis
}

\author{
JI HYE WON, MD, JI YE LEE, MD, HYUN SOOK HONG, MD, PhD and SUN HYE JEONG, MD, PhD \\ Department of Radiology, Soonchunhyang University Bucheon Hospital, Bucheon, Korea \\ Address correspondence to: Dr Ji Ye Lee \\ E-mail: peachwh@naver.com
}

Objective: To investigate the rates of thyroid nodules and cancer in pediatric cases of Hashimoto's thyroiditis (HT) in Korea.

Methods: We retrospectively reviewed 89 pediatric and adolescent patients (age, 3-18.0 years) with HT who underwent thyroid ultrasonography (US) at our institution from February 2006 to July 2016. The diagnosis of HT was based on the presence of thyroid autoantibodies. The presence of any thyroid nodules with US and cytopathologic features was analyzed. The malignancy rate was also determined.

Results: Thyroid nodules were in 20 of the 89 patients (22.4\%). Eight of these 20 patients (40\%) had colloid cysts, two (10\%) had nodular hyperplasia, one (5\%) had follicular adenoma, and two (10\%) had lymphocytic thyroiditis. Seven of the 89 patients (7.9\%) were

\section{INTRODUCTION}

Sporadic thyroid nodules are less common in children and adolescents, compared to adults, with a prevalence of 0.2 to $1.44 \%$ in children, and up to $13 \%$ in older adolescents and young adults. However, malignancy rates of thyroid nodules are 22 to $26 \%$ in children, which are higher than the rate of approximately $5 \%$ in adults. Thyroid nodules are rare among children; that said, Gupta et $\mathrm{al}^{1}$ reported a 1.6-fold higher cancer risk of sporadic thyroid nodules in children (22\%), compared to adults. ${ }^{1-4}$

Hashimoto's thyroiditis (HT), also called lymphocytic thyroiditis, is a goitrous form of autoimmune thyroiditis (AIT) and the most common thyroid disorder in children and adolescents, with a prevalence of 1.3 to $9.6 \% .{ }^{5} \mathrm{HT}$ is characterized by diffuse lymphocyte and plasma cell infiltration, fibrous replacement, and eventual atrophy of the parenchyma. According to the American Thyroid Association guidelines for children with thyroid nodules and differentiated thyroid cancer, any patient with AIT and a suspicious thyroid examination (suspected nodule, significant gland asymmetry, or palpable cervical lymphadenopathy) should confirmed to have a malignancy, all of which were papillary thyroid carcinoma (PTC); of those, five patients had diffuse sclerosing variant PTC, and two had conventional PTC on pathology.

Conclusion: The prevalence of thyroid nodules in children and adolescents with HT was $22.4 \%$. The malignancy rate of children with $\mathrm{HT}$ was $7.9 \%$. The malignancy rate among thyroid nodules was $35 \%$, which is higher than the $26 \%$ rate generally reported for children with nodules. Therefore, using thyroid US to survey known or suspected thyroid nodules might be helpful in children and adolescents with HT and may provide further useful diagnostic information.

Advances in knowledge: Thyroid US could help to assess HT patients who have known or suspected thyroid nodules. be evaluated by ultrasonography (US) with recommendations based on fair evidence. ${ }^{2}$ Dailey et $\mathrm{al}^{6}$ first reported 35 cases of malignant thyroid disease among 288 patients with AIT and suggested a positive correlation between papillary thyroid carcinoma (PTC) and HT in adults. Kim et al ${ }^{7}$ also reported a very strong correlation between PTC and $\mathrm{HT}$ in an adult population. Some reports have shown that increased anti-thyroid peroxidase antibody (anti-TPO-Ab) or high thyroid-stimulating hormone (TSH) levels are associated with an increased incidence of thyroid cancer in patients with HT. ${ }^{8,9}$

Subsequent limited adult studies have reported that the prevalence of malignant thyroid disease is 1 to $30 \%$ in patients with HT. ${ }^{10}$ Only two studies have reported an association between thyroid cancer and children and adolescents with HT. Corrias et $\mathrm{al}^{5}$ reported that the prevalence rates of thyroid nodules and thyroid cancer were 31.5 and $3.0 \%$, respectively, in a pediatric and adolescent juvenile AIT cohort. Of the thyroid nodules, 9.6\% were malignant. Keskin et $\mathrm{al}^{11}$ investigated the incidence of nodules in children with HT and reported that the thyroid nodule rate was 
$13 \%$, and the malignant thyroid cancer rate was $0.67 \% ; 5.12 \%$ of thyroid nodules were malignant. However, the prevalence of thyroid cancer among children and adolescents with HT may be different in different areas. The aim of this study was to investigate the rates of thyroid nodules and thyroid cancer in Korean children and adolescents with HT.

\section{METHODS AND MATERIALS}

\section{Patients and laboratory findings}

Approval of our institution's ethics committee was obtained for this study, and informed consent was waived due to the retrospective nature of the study. We reviewed 118 patients (range, 3-18.0 years) diagnosed with pediatric or adolescent HT who visited the Pediatric Endocrinology or Head and Neck Surgery department at our institution, with complaints of neck swelling or suspicious thyroid dysfunction (either symptoms of hypo- or hyperthyroidism) from February 2006 to July 2016. Of these, 90 children and adolescents with HT had undergone thyroid US due to neck swelling. One patient was lost to follow-up after the initial US and was excluded. The remaining 89 patients were enrolled in this study. None of the patients had a history of radiation therapy to the head and neck region.

The diagnosis of HT was based on a combination of clinical features (classic signs of hypothyroidism), and the presence of anti-TPO-Ab and thyroglobulin antibodies (Tg-Ab). Thyroid function was evaluated at the time of diagnosis as hyperthyroid, euthyroid, or hypothyroid. Laboratory data, including the serum levels of free triiodothyronine (T3, normal range 0.85-2.02 $\mathrm{mU} \mathrm{l}^{-1}$ ), free thyroxine (T4, normal range 0.93-1.71 $\mathrm{mU} \mathrm{l}^{-1}$ ), TSH (normal range, $0.27-4.2 \mathrm{mU} \mathrm{l}^{-1}$ ), anti-TPO-Ab (normal range, $0-0.3 \mathrm{U} \mathrm{ml}^{-1}$ ), and $\mathrm{Tg}-\mathrm{Ab}$ (normal range, $0-0.3 \mathrm{U} \mathrm{ml}^{-1}$ ), were retrospectively evaluated using the clinical records. Radioimmunoassays were used for laboratory examinations and were performed on the day closest to the day of the US imaging study.

\section{Analysis of US images and pathology}

US and color Doppler examinations were performed using a 5- to12-MHz linear array transducer (LAT) (IU22 US or HDI 5000; Philips Healthcare, Andover, MA), a 5- to $12-\mathrm{MHz}$ LAT (Aplio 500; Toshiba Medical Systems Corp., Nasushiobara, Japan), or a 6- to 15-MHz LAT (LOGIQ-E9; GE Healthcare, Milwaukee, WI). Patients were evaluated by thyroid US and fine-needle aspiration biopsy (FNAB) by one of two experienced faculty radiologists with 6 and 26 years of experience in thyroid imaging. The presence of any thyroid nodule was recorded. The following US features of all thyroid nodules were analyzed: (1) size; (2) number: single or multiple ( $\geq 2)$; (3) location: left or right; (4) echogenicity: anechoic, hypoechoic, isoechoic, or hyperechoic; and (5) composition: cystic, mixed, or solid. In addition to the nodule analysis, the thyroid parenchyma was analyzed for US findings of HT, including size, echogenicity, vascularity, and the presence of micronodules or septations. Since the size criterion for a thyroid nodule is problematic in children because thyroid volume changes with age, the indication for FNAB was based on the presence of suspicious features. The following suspicious US features have been suggested by Kim et al, Moon et al, and the American Thyroid Association guidelines for children with thyroid nodules and differentiated thyroid cancer: marked hypoechogenicity, irregular or microlobulated margins, increased intranodular blood flow, presence of microcalcifications, taller than wide shape. ${ }^{2,12,13}$ A thyroid nodule with at least one suspicious US finding was classified as a suspicious nodule and FNAB was performed for suspicious thyroid nodules. Three patients who had thyroid nodules without suspicious features underwent FNAB at the parents' request. FNAB specimens were categorized according to the Bethesda Thyroid Cytopathology Reporting system. ${ }^{14}$ Surgical resection was recommended for patients with Bethesda categories III-VI. Patients were classified as malignant and benign on the basis of cytological and histopathological results. Patients with colloid cysts and benign cytological results were followed up for at least 24 months.

\section{Statistical analysis}

Means and standard deviations were used to summarize continuous variables, while frequencies and percentages were used to summarize categorical variables. To compare clinical characteristics between groups, the chi-square test or Fisher's exact test was conducted for categorical variables. In the case of continuous variables, Student's $t$-test for age and the Mann-Whitney $U$ test or the Kruskal-Wallis test were used to compare levels of autoantibodies, serum TSH, and hormonal status after normality and equivalent variance testing. A two-tailed $p$-value $<0.05$ was considered significant. All statistical analyses were performed using R (v. 3.3.2, The R Foundation for Statistical Computing, Vienna, Austria).

\section{RESULTS}

\section{Patients and laboratory findings}

Subjects included 13 (14.6\%) males and 76 (85.4\%) females with ages ranging from 3 to 18 years (mean, $11.1 \pm 3.7$ years). Thyroid nodules were found in 20 (22.4\%) of the 89 children and adolescents with HT. Among 20 patients, 18 (90\%) showed nodules concomitant with the diagnosis of HT. Two patients developed thyroid nodules at 18 and 27 months after the HT diagnosis. The median follow up period was 48 months (range 17-72 months) for patients without newly developing thyroid nodules. The mean volume of all thyroid nodules was $5.54 \pm 7.59 \mathrm{~cm}^{3}$ (benign $2.53 \pm 5.6 \mathrm{~cm}^{3}$, malignant $\left.13.0 \pm 7.59 \mathrm{~cm}^{3}, p=0.006\right) .13$ patients (14.6\%) had benign nodules and seven had malignant nodules (7.9\%). Table 1 lists demographic data and levels of autoantibodies, TSH, and hormonal status according to the presence of thyroid nodules. Patients without thyroid nodules tended to be younger ( $p=0.06$ ) with higher TSH levels than patients with nodules alone $(p=0.06)$ although statistical significance was not attained. The evaluation of thyroid function at the time of diagnosis in cases with benign nodules revealed that five were euthyroid, six were subclinical hypothyroid, and two were overt hypothyroid. Among the seven patients with PTC, two were hyperthyroid, two were euthyroid, and three were subclinically hypothyroid.

\section{Analysis of US images and pathology}

Both lobes of the thyroid gland were enlarged in all patients with thyroid nodules, and various echogenicities and vascularity were detected. 
Table 1. Demographics and hormonal status according to the presence of nodules in Hashimoto's thyroiditis

\begin{tabular}{|c|c|c|c|}
\hline & $\begin{array}{c}\text { Nodule, } \\
n=20\end{array}$ & $\begin{array}{c}\text { No nodule, } \\
n=69\end{array}$ & $p^{b}$ \\
\hline Sex & $18(90 \%)$ & $58(84.1 \%)$ & 0.41 \\
\hline Female & $2(10 \%)$ & $11(15.9 \%)$ & \\
\hline Male & $12.7 \pm 2.8$ & $10.9 \pm 3.8$ & 0.06 \\
\hline Age & 100.0 & 100.0 & 0.37 \\
\hline Anti-TPOAb & \\
\hline Anti-TgAb $^{a}$ & $70.1(19.5-100.0)$ & $26.4(7.4-94.9)$ & 0.34 \\
\hline TSH $^{a}$ & $5.8(2.6-10.8)$ & $10.4(4.5-48.0)$ & 0.06 \\
\hline Hormonal status & & & 0.24 \\
\hline Euthyroidism & $7(35 \%)$ & $13(18.8 \%)$ & \\
\hline Hyperthyroidism & $2(10 \%)$ & $3(4.3 \%)$ & \\
\hline Hypothyroidism $^{a}$ & $11(55 \%)$ & $53(76.8 \%)$ & \\
\hline Echogenicity & & & 0.14 \\
\hline Diffuse low & $3(15 \%)$ & $30(43.5 \%)$ & \\
\hline Heterogenous low & $7(35 \%)$ & $13(18.8 \%)$ & \\
\hline Normal & $10(50 \%)$ & $26(37.7 \%)$ & \\
\hline Size & & & 1.00 \\
\hline Enlarged & $18(90 \%)$ & $62(89.9 \%)$ & \\
\hline Normal & $2(10 \%)$ & $7(10.1 \%)$ & \\
\hline
\end{tabular}

Anti-Tg-Ab, anti thyroglobulin antibody; Anti-TPO-Ab, anti thyroid peroxidase antibody; TSH, thyroid stimulating hormone.

Unless specified, numbers indicate patient number. Parenthesis indicated percentages of patients.

${ }^{a}$ Median value of serum level, parenthesis show 1st-3rd quartile. ${ }^{b} p<0.05$ is considered statistically significant.

Of the 20 patients with thyroid nodules, $11(11 / 20,55 \%)$ had nodules without suspicious features and nine $(9 / 20,45 \%)$ had suspicious nodules. Of the 11 nodules without suspicious features, eight $(8 / 11,72.7 \%)$ had colloid cysts on US. No new nodules or suspicious features were observed in any of the patients with

Figure 1. A 17-year-old boy with goiter. Transverse (a) and longitudinal (b) scans show heterogenous hypoechoic parenchyma. An ill-defined marked hypoechoic thyroid nodule with nonparallel orientation is seen in the left thyroid lobe. Subsequent FNAB results were lymphocytic thyroiditis. He was diagnosed at the age of 13 years. FNAB, fine-needle aspiration biopsy.

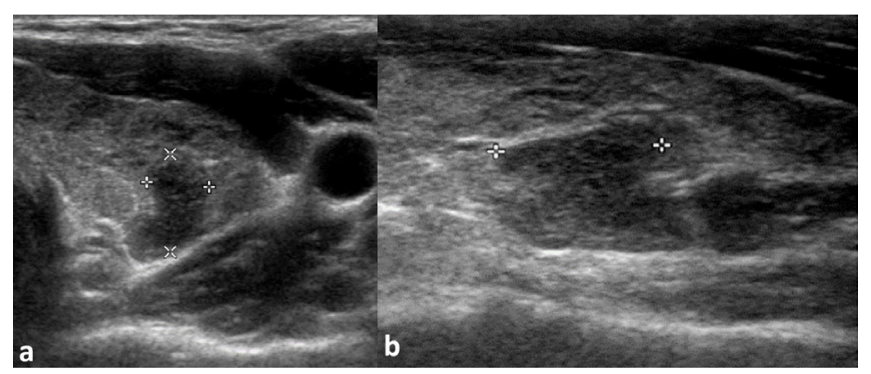

colloid cysts during follow up. FNAB was performed in three $(3 / 11,27.3 \%)$ patients with solid nodules without suspicious features because of their family history of thyroid cancer. Cytopathology revealed two cases as category II and one as category IV (suspicious for follicular neoplasm). The pathology report for the hemithyroidectomy specimen with a category IV nodule revealed follicular adenoma. Follow-up US was performed on the remaining two patients with category II nodules, and no new suspicious features or significant size increases warranted repeat biopsy.

FNAB was performed on nine patients with suspicious nodules. The results revealed category II nodules (lymphocytic thyroiditis) in two patients. One patient, diagnosed with lymphocytic thyroiditis on FNAB, underwent lobectomy for confirmatory diagnostic purposes because her thyroid gland showed asymmetrical enlargement with multiple echogenic foci, which was suspicious for microcalcifications. The surgical specimen also showed lymphocytic thyroiditis. The second case, diagnosed as lymphocytic thyroiditis on FNAB, presented with a focal ill-defined hypoechoic thyroid nodule with non-parallel orientation, which was followed up without interval change (Figure 1).

Of the remaining seven patients with suspicious features, three patients presented with focal suspicious nodules (Figure 2), and four patients presented with a diffusely enlarged gland with numerous microcalcifications (Figure 3). FNAB revealed two patients with category III (follicular lesion of undetermined significance) and five with category $\mathrm{V}$ (suspicious for malignancy; suspicious for papillary carcinoma) findings. All of the seven patients with suspicious nodules presented with suspicious lymph nodes along the lateral neck at preoperative US, and subsequent FNAB for lymph nodes was performed. Three of the seven patients showed distant (lung) metastasis at the initial presentation. Aspiration cytology of lateral neck nodes revealed metastatic papillary carcinoma in two patients with category III nodules. All seven cases (six girls and one boy) underwent surgery; all had PTC with microscopic extrathyroidal extension and lateral neck lymph node metastasis. Five patients had diffuse sclerosing variant of PTC (DSVPTC) and two patients had conventional PTC. None of the patients with thyroid cancer died during the follow up (median 38 months, range 12-64 months). All seven patients underwent radioactive iodine therapy after surgery. Four patients are on follow up without evidence of recurrence. Three patients are on follow up with residual tumors in the lung. Table 2 summarizes the clinical and imaging features of thyroid cancer. Figure 4 shows the number of patients with thyroid nodules on US and the pathological results.

\section{DISCUSSION}

In this study, the prevalence of thyroid nodules and cancer in children and adolescents with HT were 22.4 and 7.9\%, respectively. The malignancy rate among the detected thyroid nodules was $35 \%$. Among the seven patients with a malignancy, all were PTC: five patients with DSVPTC and two with conventional PTC. TSH and thyroid autoantibody levels did not differ between patients with or without thyroid nodules. 
Figure 2. A 12-year-old girl with Hashimoto's thyroiditis and papillary thyroid cancer. Transverse (a) and longitudinal (b) scan show heterogeneous echogenic nodules (arrows) with numerous microcalcifications in the upper portion of the left thyroid lobe. The lower aspect of the involved gland also shows scattered echogenic microcalcifications (c) Longitudinal scan of ipsilateral neck shows metastatic lymph nodes.

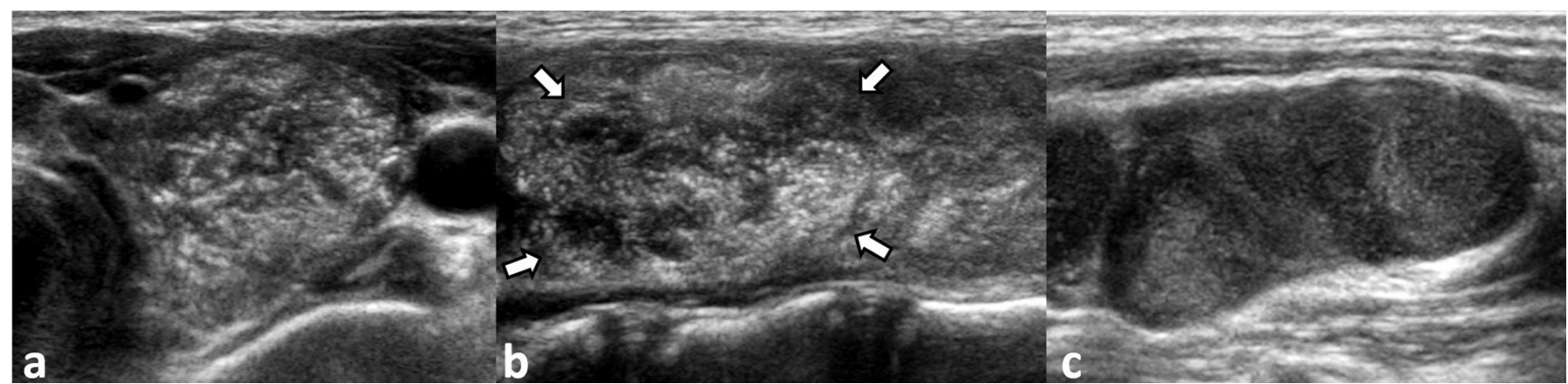

Thyroid cancer is the most common pediatric endocrine malignancy but is still rare, constituting 0.5 to $3 \%$ of all pediatric malignancies. ${ }^{15,16}$ As pediatric thyroid cancer presents with more advanced disease and a greater frequency of metastasis than in adults, these cases warrant careful evaluation and a more proactive diagnostic approach. ${ }^{17}$

Because chronic inflammation is thought to predispose a patient to neoplastic transformation and autoimmune disease, which are associated with several kinds of malignancies, ${ }^{18}$ investigators have focused on the correlation between HT and thyroid cancer since it was first described by Dailey et al. ${ }^{6}$ Some studies have reported a positive correlation, ${ }^{6,19}$ while others have failed to demonstrate an association. ${ }^{8,20,21}$

To date, few reports have been published about the prevalence of thyroid nodules and cancer in children and adolescents with HT. An Italian population had a 31.5\% (115/365) thyroid nodule prevalence, a $3.0 \%(11 / 365)$ malignancy prevalence, and a $9.6 \%(11 / 115)$ malignancy prevalence among thyroid nodules, ${ }^{5}$ whereas our subjects had a lower prevalence of thyroid nodules (22.4\%), but a higher malignancy rate (35\%). Our rates for both thyroid nodules and malignancy in patients with HT were much higher than those reported in a study by Keskin et $\mathrm{al}^{11}$ in a Turkish population; they reported a 13\% (39/300) thyroid nodule prevalence, a $0.67 \%(2 / 300)$ malignancy prevalence, and a $5.12 \%(2 / 39)$ malignancy rate among thyroid nodules.

Several hypotheses may explain these differences. First, the role of iodine status may have affected the difference in cancer rates in pediatric HT. Iodine deficiency is well known to be a risk factor for thyroid nodules and the follicular histological type of thyroid cancer. ${ }^{22}$ However, the association between PTC and excessive iodine intake remain unclear. One study suggested that high iodine uptake may increase the risk of the papillary thyroid cancer. ${ }^{23,24}$ Italy and Turkey are countries with mild to moderate iodine deficiency, whereas South Korea is an iodine-sufficient region. ${ }^{25}$ Because of the limitations of the retrospective design, this was difficult to assess, but a difference in iodine status may have influenced the prevalence of thyroid nodules and malignancy. Second, the role of TSH should be discussed. There has long been a controversy in the literature about a positive correlation between HT and PTC. Kim et $\mathrm{al}^{26}$ reported a very strong correlation between PTC and HT, and proposed Tg-Ab as an independent risk factor for thyroid cancer. Many previous studies have indicated that higher serum TSH concentrations

Figure 3. A 18-year-old girl with Hashimoto's thyroiditis and papillary thyroid cancer. Transverse (a) and longitudinal image (b) shows diffuse enlargement of both thyroid lobes with scattered innumerable microcalcifications.

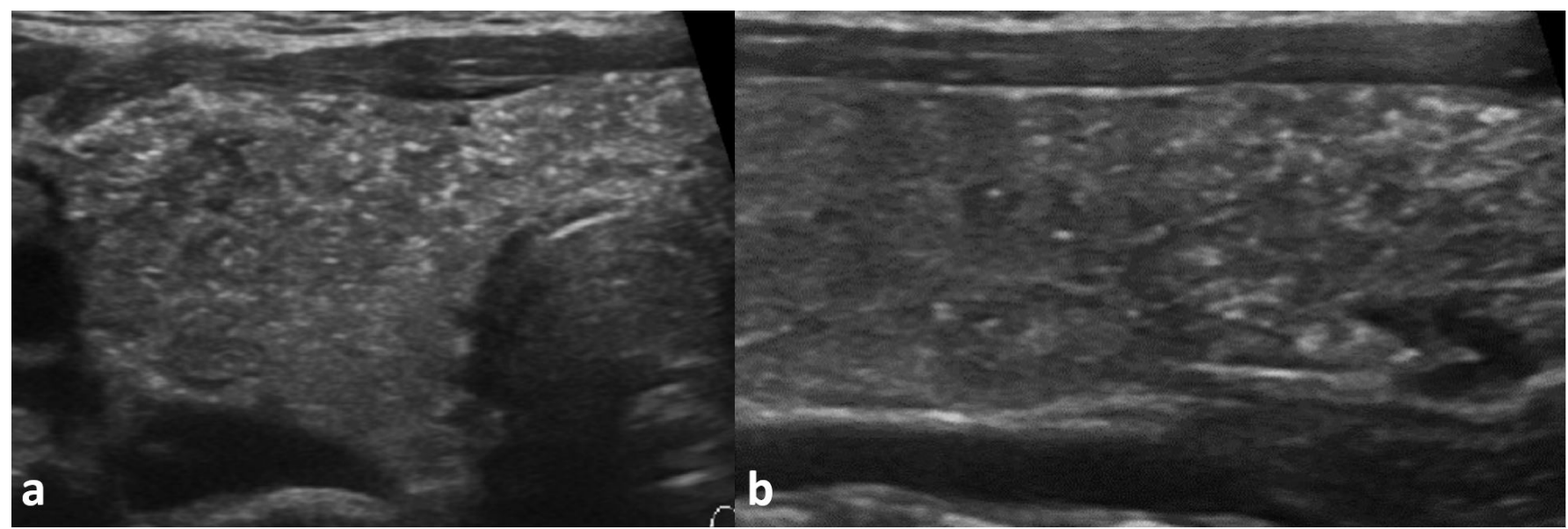




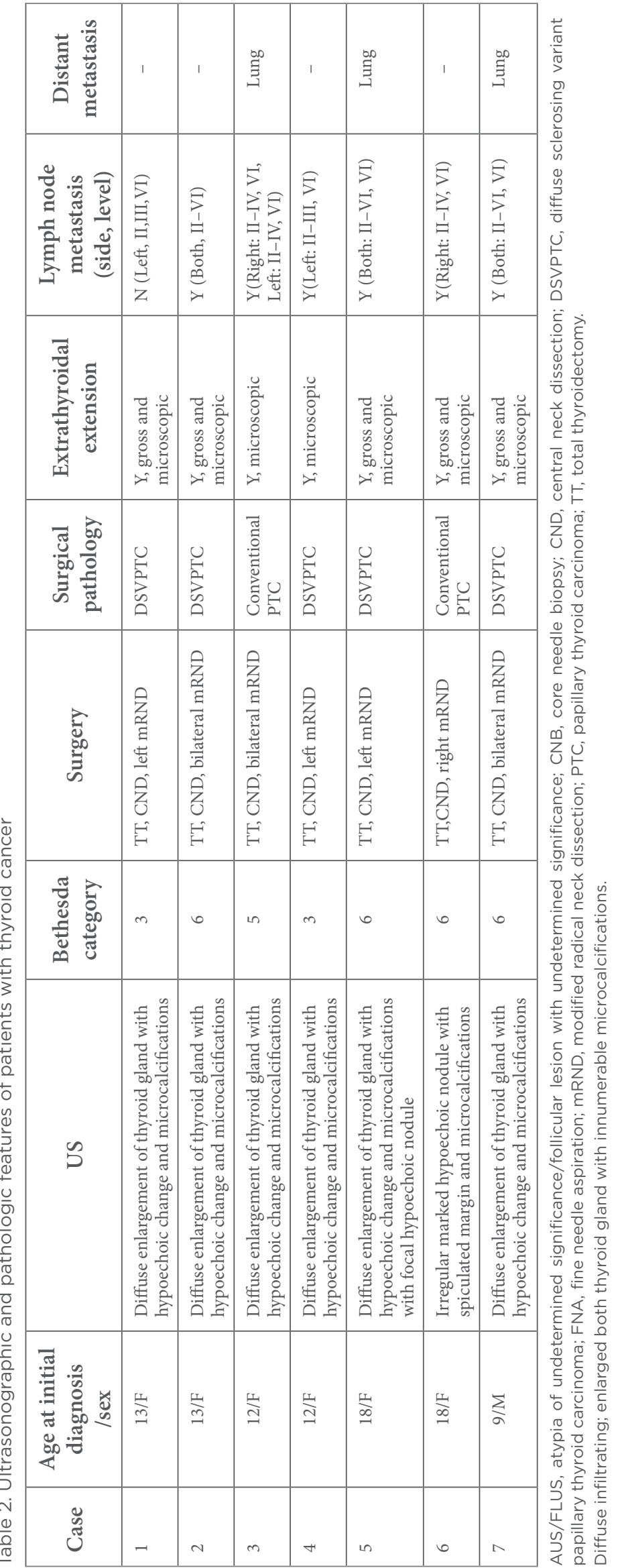


Figure 4. Flow chart of nodule characteristics and results of cytopathology. US, ultrasonography; FNAB, fine-needle aspiration biopsy; FLUS, follicular lesion of undetermined significance; PTC, papillary thyroid carcinoma; DSVPTC, diffuse sclerosing variant of papillary thyroid carcinoma.

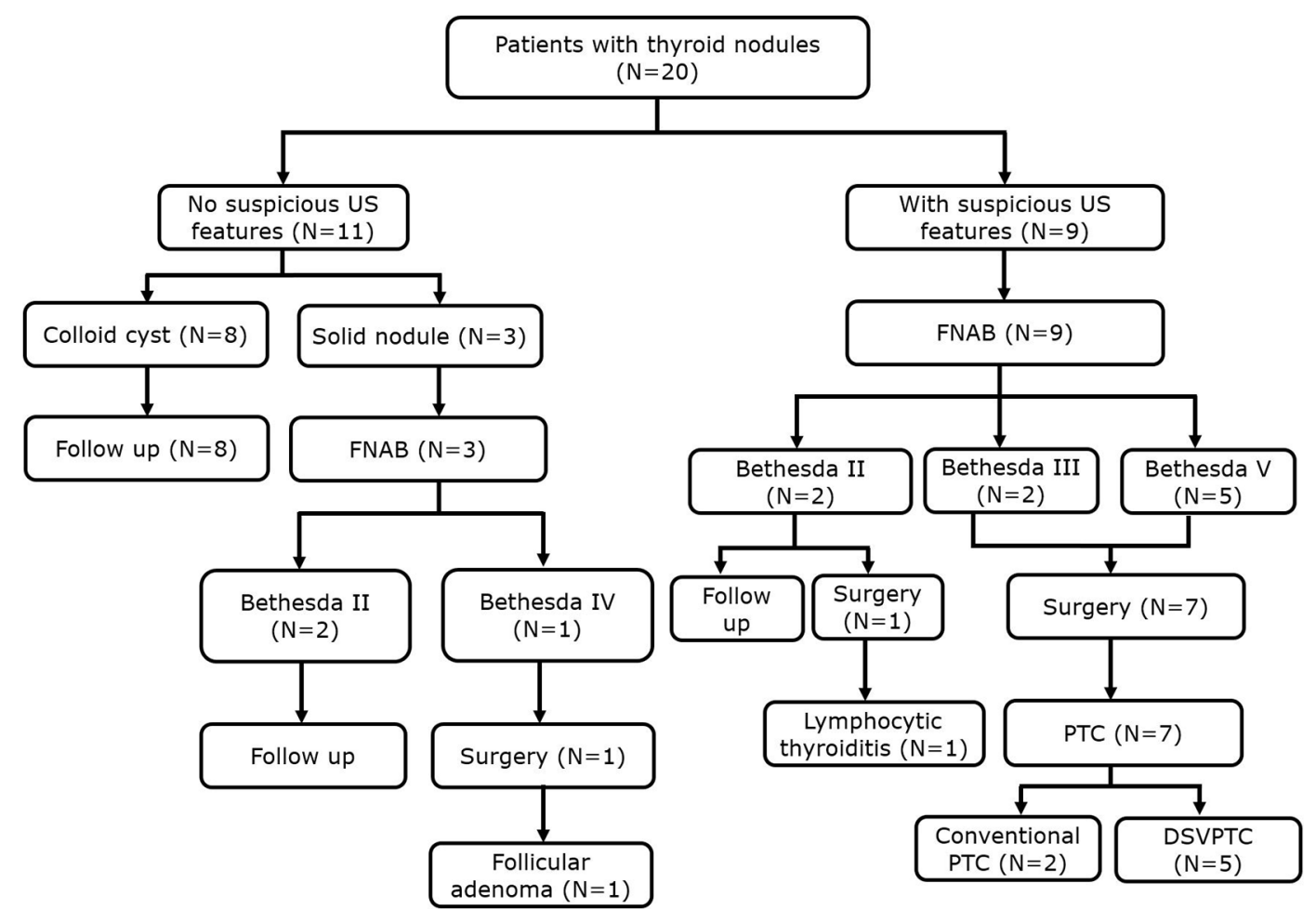

are associated with an increased frequency of benign and malignant thyroid nodules in patients without AIT. ${ }^{8,9,27}$ A study by Fiore et al also showed that the risk of thyroid cancer increases when serum TSH was high, regardless of thyroid autoantibody status. $^{28}$ The prevalence of malignancy in this retrospective study was much higher (35\%) than the 5 to $15 \%$ malignancy risk expected for thyroid nodules. ${ }^{29}$ In this study, patients with thyroid nodules had lower TSH levels than HT patients without thyroid nodules and there were no significant differences in the levels of autoantibodies observed between the patient groups. Our results contradict many previous studies, which may indicate that the mechanisms underlying the relationship between increased serum TSH and thyroid cancer are different from those affecting the link between AIT and thyroid malignancy. However in our study, TSH levels obtained from patients with HT and data of comparable healthy controls were not available. Small patient numbers may also partly explain this contradiction with previous studies.

In similar studies of Italian and Turkish populations, ${ }^{5,11}$ the most common type of HT merger thyroid cancer was PTC, but further histological subtypes were not described. In this study, all seven patients with thyroid cancer had PTC: five had DSVPTC and two had conventional PTC. Among the five patients with DSVPTC, four presented with the diffuse infiltrating form of PTC, and the other presented with nodular lesions. Even though the patient presented with nodular lesions, the remaining thyroid parenchyma had numerous microcalcifications. To date, there is no established relationship between HT and DSVPTC, so additional studies are required. Molecular biological studies about DSVPTC suggest that it exhibits different expression patterns of galectin 3 , epithelial membrane antigen, p53, p64, and cell adhesion molecules compared to conventional PTC. A genetic analysis revealed that activation of rearranged during transfection (RET)/ PTC rearrangements is common in DSVPTC with a lack of $\mathrm{BRAF}^{\mathrm{V} 600 \mathrm{E}}$ and RAS mutations. ${ }^{30,31}$ The association between HT and thyroid cancer is not well understood, but several authors have suggested the role of RET/PTC rearrangement in the association between PTC and HT. Previous studies have suggested that chronic inflammation might facilitate the RET/PTC rearrangement, or vice versa. ${ }^{32-34}$ Additionally, Muzza et $\mathrm{al}^{35}$ and Kim et $\mathrm{al}^{36}$ reported that the $\mathrm{BRAF}{ }^{\mathrm{V} 600 \mathrm{E}}$ mutation, a common genetic alteration, is associated with a lower frequency of PTC in the background of HT. Therefore, we presumed that these common genetic features of increased RET/PTC rearrangement and fewer $\mathrm{BRAF}^{\mathrm{V} 600 \mathrm{E}}$ mutations might be a link between DSVPTC and HT. However, molecular studies were not performed in our study, so further studies are needed to validate this hypothesis.

This study had several limitations. First, it included only a small number of HT merger malignant thyroid disease cases. In fact, this limitation arises from the significantly lower incidence of HT in children. Therefore, a larger series must be conducted to verify our results. Second, our analysis was retrospective, and the study population was limited to patients with HT who had US examinations at a single institution, which could lead to selection biases. Third, FNAB was not performed on colloid cysts. Two patients with benign FNAB results did not undergo 
a surgical biopsy. However, patients who did not undergo FNAB and patients with benign cytological results were followed up for at least 24 months without changes; therefore, we believe our nodular prevalence well reflects the actual incidence.

\section{CONCLUSION}

The prevalence of thyroid nodules and malignancy were 22.4 and $7.9 \%$, respectively, in children and adolescents with HT. The malignancy rate among thyroid nodules was $35 \%$. The malignancy risk of thyroid nodules developing in an HT background is higher than the $26 \%$ rate generally reported for children with nodules. All patients with thyroid cancer presented with advanced disease. Specifically, $71.4 \%$ of patients with thyroid cancer had DSVPTC. Therefore, clinicians should be aware of the high malignancy risk of thyroid nodules in children and adolescents with HT, and US might be helpful to survey known or suspected thyroid nodules in these patients.

\section{COMPETING INTERESTS}

The authors of this manuscript declare no relationships with any companies, whose products or services may be related to the subject matter of the article.

\section{FUNDING}

This study was supported in part by the Soonchunhyang University Research Fund.

\section{ETHICS APPROVAL}

Institutional Review Board of Soonchunhyang University Bucheon Hospital approved this study (2017-08-009).

\section{PATIENT CONSENT}

Written informed consent was waived by the Institutional Review Board.

\section{REFERENCES}

1. Gupta A, Ly S, Castroneves LA, Frates MC, Benson CB, Feldman HA, et al. A standardized assessment of thyroid nodules in children confirms higher cancer prevalence than in adults. J Clin Endocrinol Metab 2013; 98: 3238-45. doi: https://doi. org/10.1210/jc.2013-1796

2. Francis GL, Waguespack SG, Bauer AJ, Angelos P, Benvenga S, Cerutti JM, et al. Management guidelines for children with thyroid nodules and differentiated thyroid cancer. Thyroid 2015; 25: 716-59. doi: https:// doi.org/10.1089/thy.2014.0460

3. Millman B, Pelutteri PK. Nodular thyroid disease in children and adolescents. Otolaryngol Head Neck Surg 1997; 116: 604-9. doi: https://doi.org/10.1016/S01945998(97)70235-3

4. Niedziela M. Pathogenesis, diagnosis and management of thyroid nodules in children. Endocr Relat Cancer 2006; 13: 427-53. doi: https://doi.org/10.1677/erc.1.00882

5. Corrias A, Cassio A, Weber G, Mussa A, Wasniewska M, Rapa A, et al. Thyroid nodules and cancer in children and adolescents affected by autoimmune thyroiditis. Arch Pediatr Adolesc Med 2008; 162: 526-31. doi: https://doi.org/10.1001/ archpedi.162.6.526

6. Dailey ME, Lindsay S, Skahen R. Relation of thyroid neoplasms to hashimoto disease of the thyroid gland. AMA Arch Surg 1955; 70: 291-7. doi: https://doi.org/10.1001/archsurg. 1955.01270080137023

7. Kim KW, Park YJ, Kim EH, Park SY, Park DJ, Ahn SH, et al. Elevated risk of papillary thyroid cancer in Korean patients with hashimoto's thyroiditis. Head Neck 2011;
33: 691-5. doi: https://doi.org/10.1002/hed. 21518

8. Haymart MR, Repplinger DJ, Leverson GE, Elson DF, Sippel RS, Jaume JC, et al. Higher serum thyroid stimulating hormone level in thyroid nodule patients is associated with greater risks of differentiated thyroid cancer and advanced tumor stage. J Clin Endocrinol Metab 2008; 93: 809-14. doi: https://doi.org/ 10.1210/jc. 2007-2215

9. Boelaert K, Horacek J, Holder RL, Watkinson JC, Sheppard MC, Franklyn JA. Serum thyrotropin concentration as a novel predictor of malignancy in thyroid nodules investigated by fine-needle aspiration. J Clin Endocrinol Metab 2006; 91: 4295-301. doi: https://doi.org/10.1210/jc.2006-0527

10. Carson HJ, Castelli MJ, Gattuso P. Incidence of neoplasia in hashimoto's thyroiditis: a fineneedle aspiration study. Diagn Cytopathol 1996; 14: 38-42. doi: https://doi.org/10.1002/ (SICI)1097-0339(199602)14:1<38::AIDDC8>3.0.CO;2-R

11. Keskin M, Savas-Erdeve S, Aycan Z. Coexistence of thyroid nodule and thyroid cancer in children and adolescents with hashimoto thyroiditis: a single-center study. Horm Res Paediatr 2016; 85: 181-7. doi: https://doi.org/10.1159/000443143

12. Kim EK, Park CS, Chung WY, Oh KK, Kim DI, Lee JT, et al. New sonographic criteria for recommending fine-needle aspiration biopsy of nonpalpable solid nodules of the thyroid. AJR Am J Roentgenol 2002; 178: 687-91. doi: https://doi.org/10.2214/ajr. 178.3.1780687

13. Moon WJ, Jung SL, Lee JH, Na DG, Baek JH, Lee $\mathrm{YH}$, et al. Benign and malignant thyroid nodules: US differentiation-multicenter retrospective study. Radiology 2008; 247: 762-70. doi: https://doi.org/10.1148/radiol. 2473070944

14. Cibas ES, Ali SZ, NCI Thyroid FNA State of the Science Conference. The bethesda system for reporting thyroid cytopathology. Am J Clin Pathol 2009; 132: 658-65. doi: https://doi.org/10.1309/ AJCPPHLWMI3JV4LA

15. Millman B, Pellitteri PK. Thyroid carcinoma in children and adolescents. Arch Otolaryngol Head Neck Surg 1995; 121: 1261-4. doi: https://doi.org/10.1001/archotol.1995. 01890110037007

16. Shapiro NL, Bhattacharyya N. Populationbased outcomes for pediatric thyroid carcinoma. Laryngoscope 2005; 115 : 337-40. doi: https://doi.org/10.1097/01.mlg. 0000154743.71184 .09

17. Zimmerman D, Hay ID, Gough IR, Goellner JR, Ryan JJ, Grant CS, et al. Papillary thyroid carcinoma in children and adults: long-term follow-up of 1039 patients conservatively treated at one institution during three decades. Surgery 1988; 104: 1157-66.

18. Bernatsky S, Ramsey-Goldman R, Clarke A. Malignancy and autoimmunity. Curr Opin Rheumatol 2006; 18: 129-34. doi: https://doi. org/10.1097/01.bor.0000209423.39033.94

19. Okayasu I, Fujiwara M, Hara Y, Tanaka Y, Rose NR. Association of chronic lymphocytic thyroiditis and thyroid papillary carcinoma. A study of surgical cases among Japanese, and white and African Americans. Cancer 1995; 76: 2312-8. doi: https://doi.org/10. 1002/1097-0142(19951201)76:11<2312::AIDCNCR2820761120>3.0.CO;2-H 
20. Holm LE, Blomgren $\mathrm{H}$, Löwhagen $\mathrm{T}$. Cancer risks in patients with chronic lymphocytic thyroiditis. N Engl J Med 1985; 312: 601-4. doi: https://doi.org/10.1056/ NEJM198503073121001

21. Rago T, Di Coscio G, Ugolini C, Scutari M, Basolo F, Latrofa F, et al. Clinical features of thyroid autoimmunity are associated with thyroiditis on histology and are not predictive of malignancy in 570 patients with indeterminate nodules on cytology who had a thyroidectomy. Clin Endocrinol 2007; 67: 363-9. doi: https://doi.org/10.1111/j.13652265.2007.02892.x

22. Zimmermann MB, Galetti V. Iodine intake as a risk factor for thyroid cancer: a comprehensive review of animal and human studies. Thyroid Res 2015; 8: 8. doi: https:// doi.org/10.1186/s13044-015-0020-8

23. Knobel M, Medeiros-Neto G. Relevance of iodine intake as a reputed predisposing factor for thyroid cancer. Arq Bras Endocrinol Metabol 2007; 51: 701-12. doi: https://doi. org/10.1590/S0004-27302007000500007

24. Peterson E, De P, Nuttall R. BMI, diet and female reproductive factors as risks for thyroid cancer: a systematic review. PLoS One 2012; 7: e29177. doi: https://doi.org/10. 1371/journal.pone.0029177

25. Andersson M, Karumbunathan V, Zimmermann MB. Global iodine status in 2011 and trends over the past decade. J Nutr 2012; 142: 744-50. doi: https://doi.org/10. 3945/jn.111.149393

26. Kim ES, Lim DJ, Baek KH, Lee JM, Kim MK, Kwon HS, et al. Thyroglobulin antibody is associated with increased cancer risk in thyroid nodules. Thyroid 2010; 20: 885-91. doi: https://doi.org/10.1089/thy.2009.0384

27. Mussa A, De Andrea M, Motta M, Mormile A, Palestini N, Corrias A. Predictors of malignancy in children with thyroid nodules. J Pediatr 2015; 167: 886-92. doi: https://doi. org/10.1016/j.jpeds.2015.06.026

28. Fiore E, Rago T, Latrofa F, Provenzale MA, Piaggi P, Delitala A, et al. Hashimoto's thyroiditis is associated with papillary thyroid carcinoma: role of TSH and of treatment with L-thyroxine. Endocr Relat Cancer 2011; 18: 429-37. doi: https://doi.org/ 10.1530/ERC-11-0028

29. Haugen BR, Alexander EK, Bible KC, Doherty GM, Mandel SJ, Nikiforov YE, et al. 2015 American thyroid association management guidelines for adult patients with thyroid nodules and differentiated thyroid cancer: the American thyroid association guidelines task force on thyroid nodules and differentiated thyroid cancer. Thyroid 2016; 26: 1-133. doi: https://doi.org/ 10.1089/thy.2015.0020

30. Sheu SY, Schwertheim S, Worm K, Grabellus F, Schmid KW. Diffuse sclerosing variant of papillary thyroid carcinoma: lack of BRAF mutation but occurrence of RET/ PTC rearrangements. Mod Pathol 2007; 20: 779-87. doi: https://doi.org/10.1038/ modpathol.3800797

31. Pillai S, Gopalan V, Smith RA, Lam AK. Diffuse sclerosing variant of papillary thyroid carcinoma-an update of its clinicopathological features and molecular biology. Crit Rev Oncol Hematol 2015; 94: 64-73. doi: https://doi.org/10.1016/j. critrevonc.2014.12.001

32. Colotta F, Allavena P, Sica A, Garlanda C, Mantovani A. Cancer-related inflammation, the seventh hallmark of cancer: links to genetic instability. Carcinogenesis 2009; 30: 1073-81. doi: https://doi.org/10.1093/carcin/ bgp 127

33. Guarino V, Castellone MD, Avilla E, Melillo RM. Thyroid cancer and inflammation. Mol Cell Endocrinol 2010; 321: 94-102. doi: https://doi.org/10.1016/j.mce.2009.10.003

34. Kang DY, Kim KH, Kim JM, Kim SH, Kim JY, Baik HW, et al. High prevalence of RET, RAS, and ERK expression in Hashimoto's thyroiditis and in papillary thyroid carcinoma in the Korean population. Thyroid 2007; 17: 1031-7. doi: https://doi.org/10. 1089/thy.2007.0035

35. Muzza M, Degl'Innocenti D, Colombo C, Perrino M, Ravasi E, Rossi S, et al. The tight relationship between papillary thyroid cancer, autoimmunity and inflammation: clinical and molecular studies. Clin Endocrinol 2010; 72: 702-8. doi: https://doi. org/10.1111/j.1365-2265.2009.03699.x

36. Kim SK, Song KH, Lim SD, Lim YC, Yoo YB, Kim JS, et al. Clinical and pathological features and the BRAF(V600E) mutation in patients with papillary thyroid carcinoma with and without concurrent Hashimoto thyroiditis. Thyroid 2009; 19: 137-41. doi: https://doi.org/10.1089/thy.2008.0144 